\title{
Steric Crowding Effects on Target Detection in an Affinity Biosensor
}

\author{
Lisa Bonanno ${ }^{a}$ and Lisa DeLouise ${ }^{a, b, *}$ \\ aDepartment of Biomedical Engineering, University of Rochester School of Medicine and Dentistry, 601 \\ Elmwood Ave., Rochester, NY 14642, USA \\ $\mathrm{b}$ Department of Dermatology, University of Rochester School of Medicine and Dentistry, 601 Elmwood Ave., \\ Rochester, NY 14642, USA
}

\section{Abstract}

This work quantifies the impact of steric crowding on whole antibody $(\mathrm{Ab})$ receptor immobilization and target $\mathrm{Ab}$ detection and also demonstrates how the versatile biotin/streptavidin receptor immobilization system must be tuned to optimize target detection in designing biosensors. Results are demonstrated on a label-free optical biosensor comprised of n-type macroporous porous silicon (PSi) with $\sim 88-107 \mathrm{~nm}$ diameter pores. We employ a sandwich assay scheme comprised of a linking chemistry (biotin/streptavidin) to attach biotinylated anti-rabbit IgG (receptor) to detect rabbit IgG (target). A "bottoms-up" approach was taken to investigate each layer of the sandwich assay to optimize target binding. Steric crowding was observed to hinder subsequent layer binding for each layer in the sandwich (biotin, streptavidin, and receptor). Our results give definitive evidence that onset of steric crowding within the biotin layer occurs at a surface coverage of 57\% which is much higher compared to published work on well ordered self-assembled biotin monolayers on planar gold surfaces. This difference is attributed to the topographical heterogeneity of the PSi substrate. Streptavidin binding to surface-linked biotin was altered by preblocking streptavidin binding sites with biotin. Through consistent trends in data, preblocking SA was shown to reduce steric crowding within the SA layer, which translated into increased receptor immobilization. The final detection range of rabbit IgG was $0.07-3 \mathrm{mg} \mathrm{ml}^{-1}\left(0.23-9.8 \mu \mathrm{g} \mathrm{mm}^{-2}\right)$ and binding specificity was demonstrated employing anti-chicken IgG control receptor. This study underlines the importance of considering binding avidity and surface topography in optimizing chip-based biosensors.

\section{Introduction}

Streptavidin (SA) and biotin are commonly used in many biotechnological applications due to their high binding affinity $\left(\mathrm{K}_{\mathrm{d}} \sim 10^{-15} \mathrm{M}\right) .{ }^{1}$ The SA-biotin link is commonly employed in biodetection, 2,3 recombinant protein purification, ${ }^{4}$ and fluorophore tagging 5 . It is also one of the most common receptor immobilization chemistries used in biosensor development. ${ }^{6} \mathrm{In}$ this paper we utilize this linking system to characterize and optimize the performance of a macroporous silicon optical biosensor. Equilibrium theory predicts that more target molecules can be captured by raising the immobilized receptor concentration. ${ }^{7}$ This relationship can be advantageous for low binding affinity systems and is exploited by nature in a variety of biological processes. For example, cells have developed the ability to mobilize high concentrations of cytosolic adaptor proteins and enzymes in a small volume near the cellular membrane to achieve efficient signaling. 8 Other natural processes that illustrate the positive

\footnotetext{
*To whom correspondence should be addressed. Department of Dermatology, University of Rochester School of Medicine and Dentistry, Box 697, 601 Elmwood Ave., Rochester, NY 14642 USA. Tel: +1-585-275-1810; E-mail address: lisa_delouise@urmc.rochester.edu
} 
utilization of high density binding to a specific target are cell signaling through integrin clustering ${ }^{9}$ and carbohydrate multivalent binding to a receptor ${ }^{10}$. In certain cases steric crowding may have negative effects such as causing protein misfolding and aggregation which is a cause of human motor neuron disease. ${ }^{11}$

In contrast to the biological systems discussed most biosensor platforms, as well as the one described in this paper, immobilize receptors to a solid surface which is more rigid than the cellular environment. Therefore receptors have less ability to optimize steric crowding which can impede target binding and; therefore, decrease biosensor sensitivity. ${ }^{12,13,14}$ This is a particular concern in multivalent linking systems like SA which has four equivalent biotinbinding sites per molecule. This negative effect has been lessened by the use of long linking molecules to extend the distance between the immobilized receptor and sensor surface.

The binding and dissociation of wild-type and mutant SA on immobilized biotin has been characterized and modeled on planar self-assembled monolayers on gold surfaces ${ }^{12,13}$. It was characterized by Pérez-Luna et al. ${ }^{13}$ and with more detail by Jung et al. ${ }^{12}$ that at low biotin surface coverage $(<10 \%)$ and high biotin surface coverage $(>60 \%)$ SA binds via a single biotin-binding site. Over a median range of biotin surface coverage (10-40\%) mutant streptavidin exhibited second order off rate constants from which they concluded SA binds mainly via two biotin-binding sites. It was also reported in both studies above that at biotin surface coverage higher than $20 \%$ the total amount of bound SA decreases. This was attributed to steric crowding in the biotin layer in which tightly packed surface-bound biotin inhibits the proper orientation of the SA biotin-binding pocket. These studies provide significant insight into the complexity of SA/biotin linking chemistry on idealized planar surfaces. They also provide a foundation for comparing our results while investigating the development of this linking system on topographically heterogeneous porous silicon substrates and in particular quantifying its effect on biosensor detection sensitivity.

The morphology of porous silicon (PSi) has been well studied and its applications have emerged in diverse fields such as solar cells, ${ }^{15}$ fuel cells,,${ }^{16}$ and drug delivery. ${ }^{17}$ Many groups have proved the use of PSi as an optical biosensing substrate. ${ }^{2,18,19,20}$ The amount of biological material linkedto the internal surface area causes a decrease in the porosity of the porous device thatcan be monitored by a shift in refractive index. Porosity can be related to refractive index by the Bruggeman effective medium approximation. ${ }^{18,21}$ The effective refractive index $(\eta)$ of the material is a function of the void volume $\left(\eta_{\text {air }}=1\right)$, silicon substrate $\left(\eta_{\mathrm{Si}}=3.4\right)$, and the index of refraction of substances that link to the internal surface area. The observed wavelengthshift $(\Delta \lambda)$ is a function of the index of refraction of the bound material and the thickness of the bound layer; which is referred to as the optical pathlength ${ }^{18}$. A red shift of the optical reflectance spectrum towards longer wavelengths results from an increase in the optical path length. The wavelength shift sensitivity (WSS) of a microcavity is characterized by Equation 1. Studies have shown WSS depends on the frequency at which measurements are made. ${ }^{7}$ The sensitivity of the sensors used in this study is $\Delta \lambda / \Delta \eta \sim 140 \mathrm{~nm}$ $\mathrm{RI}^{-1}$ at a measuring wavelength of $\sim 750 \mathrm{~nm}$, which is within the optimal spectral range of the spectrometer used in these experiments.

$$
\text { Sensitivity }=\frac{\Delta \lambda}{\Delta \eta}
$$

This paper builds on an initial study recently reported by Ouyang et al ${ }^{19}$ that demonstrated a proof-of-principle that macroporous PSi microcavity sensors can be used for immuno-based sandwich assays. In contrast to mesoporous devices (2-50 $\mathrm{nm}$ pore diameters), the morphology of macroporous silicon is sufficiently large to enable pore infiltration and stacking of immunoglobulin ( $\operatorname{IgG}$ radius $\sim 5.6 \mathrm{~nm}^{22}$ ) within the porous matrix. They employed the biotinSA linking system as a proof of principle illustrating the capability of $\mathrm{a}^{+}$macroporous 
microcavity to act as a biosensing device. A decrease in the ability to bind streptavidin was observed for surface concentrations of biotin above $50 \%$ and was attributed to steric crowding within the biotin layer. ${ }^{19}$ In this study we leverage the optical response of the PSi sensor to directly investigate each layer of a sandwich assay which attaches a biotinylated receptor antibody to the biotin/SA linking chemistry in order to optimize target detection. Taking a "bottom-up" approach we observe strong steric crowding effects in each layer of the receptor linking stack (biotin/SA/receptor) and identify distinct differences when compared to results reported on planar gold surfaces. We also investigate whether the effects of steric crowding can be overcome to enhance target detection sensitivity by decreasing avidity through preblocking biotin-binding sites on SA. Our results are of general interest because many biotechnological applications such as ELISA, recombinant protein purifications, and column chromatography methods employ the versatile biotin-SA link and also have topographically heterogeneous substrates.

\section{Experimental Section}

\subsection{Biosensor Fabrication}

The starting material in the sensor fabrication process was n-type silicon wafers with <100> orientation and Sb dopant (SEH America, Inc.). The resistivity range was 0.007-0.02 ohm-cm. An electrolyte solution consisting of $5 \%$ Hydrofluoric acid (HF) and $0.1 \%$ Pluronic L31 (BASF) surfactant was used to etch the porous silicon. One dimensional photonic bandgap microcavity structures were etched into the silicon substrate in a custom designed Teflon cell at room temperature. ${ }^{18}$ During etching the electrolyte was gently mixed using a manual pipette pump. After etching was complete the electrolyte was rinsed off using ethanol, then water and dried under a stream of nitrogen gas. The etching process produced a porous silicon device with planar diameter of approximately $1.4 \mathrm{~cm}$ (Refer to Fig 1 (b) inset); which was diced evenly into six individual porous silicon sensors.

The microcavity structure employed in this study consisted of two Bragg mirrors separated by a half-wavelength optical thickness layer which allows for destructive interference to form a resonance wavelength $(\sim 750 \mathrm{~nm})$ for which there is low reflectance (high transmission). ${ }^{18}$ Figure 1 displays a SEM side view image of a microcavity and its optical reflectance spectrum. Prior to etching the microcavity structure a sacrificial layer was etched at a current density $(\mathrm{J})$ of $60 \mathrm{~mA} \mathrm{~cm}^{-2}$ for $30 \mathrm{~s}$, followed by 2 short current pulses ( $\mathrm{J}=300 \mathrm{~mA} \mathrm{~cm}{ }^{-2}$ for $\left.1.5 \mathrm{~s}\right)$ to electropolish off the sacrificial layer. This creates defects on the Si surface to seed pore formation in highly doped n-type Si where holes are the minority carrier. ${ }^{19}$ High and low refractive index layers of the microcavity were etched at $30 \mathrm{~mA} \mathrm{~cm}^{-2}$ for $3.23 \mathrm{~s}$ and $60 \mathrm{~mA}$ $\mathrm{cm}^{-2}$ for $2.87 \mathrm{~s}$ respectively and the half-wavelength optical thickness layer was formed with a current density of $60 \mathrm{~mA} \mathrm{~cm}^{-2}$ for $5.74 \mathrm{~s}$. Gravimetric measurements made on these samples indicated porosities of $64 \%$ and $77 \%$ for the high and low refractive index layers. SEM micrograph analysis confirmed the pore diameters to range from $88.2 \pm 39.3 \mathrm{~nm}$ to $106.9 \pm 40.8$ $\mathrm{nm}$ for the high and low refractive index layers and the depth of the microcavity structure was approximately $4.0 \mu \mathrm{m}$. Interface roughness and the small refractive index contrast between high and low porosity layers results in a quality factor $\left(\mathrm{Q}=\lambda_{\mathrm{r}} / \Delta \lambda\right)$ of $\sim 66.5$ (Fig 1$)$. It is important to note that sensitivity of the microcavity device is independent of $\mathrm{Q}^{7}$ and therefore the contrast between layers was minimized to ensure adequate infiltration of antibodies through the porous device. Single layer interferometric films could have also been used in this experiment $^{23,24}$, however the pattern of a microcavity optical reflectance spectrum with its characteristic resonance wavelength dip allows for ease in monitoring optical shifts. 


\subsection{Thermal Oxidation}

Macroporous silicon is thermally oxidized to impart greater stability in biological solutions containing salt and to create hydrophilic pore channels. ${ }^{19}$ Dry thermal oxidation was conducted using a three-zone Lindberg tube furnace at $900^{\circ} \mathrm{C}$. Samples were slowly introduced into the center zone of the furnace where annealing took place for $3 \mathrm{~min}$. The entire oxidation cycle is completed over a 15 min period.

\subsection{Linking Chemistry}

Figure 2 is an illustration depicting the sandwich assay used in this experiment and discussed in sections 2.3-2.4. All concentrations cited in this paper refer to solution concentrations applied to the sensor surface unless otherwise stated.

Amino Silanization-After oxidation the internal surface of the porous silicon was silanized with $2 \%$ aqueous 3-aminopropyltrimethoxysilane (APTMS, Gelest) mixed in $50 \%$ ethanol for $15 \mathrm{~min}$. The chips were rinsed with ethanol, then water and dried in a stream of nitrogen gas. The sensors were then kept at $100{ }^{\circ} \mathrm{C}$ for $10 \mathrm{~min}$ to evaporate any remaining solvents and to form crosslinks in the silane layer. A red shift of the optical reflectance spectrum of approximately $3 \mathrm{~nm}$ was measured due to the APTMS indicating saturation coverage. APTMS forms a monolayer with a thickness of $8.7 \pm 0.5 \AA$ as determined by ellipsometry. ${ }^{25}$

NHS-Linked Biotin and Streptavidin-Amine reactive sulfo-NHS-LC-LC-biotin (sulfosuccinimidyl-6-(biotinamido)-6-hexanamido hexanoate, 669.75 Da, Pierce), was diluted in phosphate buffered saline (PBS) buffer $(\mathrm{pH}=7.4)$ and used immediately. Different concentrations were studied ranging from $0-2 \mathrm{mg} \mathrm{ml}^{-1}$. Biotin was immobilized to the amineterminated porous silicon surface by adding $15 \mu \mathrm{l}$ of the desired concentration of sulfo-NHSLC-LC-biotin on the sensor surface by pipette and left for $30 \mathrm{~min}$ in a humidified enclosure to prevent evaporation. The solution was rinsed off with PBS buffer and dried under a stream of nitrogen gas. A water rinse was not used.

Immunopure streptavidin ( $53 \mathrm{kDa}$, Pierce) was diluted in $20 \mathrm{mM}$ potassium phosphate buffer (pH 6.5) to the desired concentration ranging between $0-0.5 \mathrm{mg} \mathrm{ml}^{-1}$. The sensor surface was functionalized with streptavidin by applying $15 \mu \mathrm{l}$ of the diluted sample for $45 \mathrm{~min}$ in a humidified enclosure. The streptavidin solution was rinsed off with PBS buffer and dried under a stream of nitrogen gas.

\subsection{Receptor and Target Chemistry}

Unless otherwise stated all materials were purchased from Sigma-Aldrich and used without further purification. The receptor molecule was biotinylated whole anti-rabbit $\operatorname{IgG}(\mathrm{H} \& \mathrm{~L})$ from a goat host. The biotinylated antibody (B-Ab) receptor was diluted in PBS buffer and $15 \mu \mathrm{l}$ of solution was soaked on the sensor within a humidified enclosure for 1 hour to allow diffusion of antibody through the porous matrix. A concentration range from $0-1 \mathrm{mg} \mathrm{ml}^{-1}$ was studied. The B-Ab solution was rinsed with PBS buffer and soaked in $5 \mathrm{ml}$ of PBS buffer for $5 \mathrm{~min}$ to reduce weak non-specific binding of the B-Ab. Before drying the sensors with nitrogen gas, they were rinsed with $15 \mu \mathrm{l}$ of preservation solution containing $1 \%$ trehalose and $0.1 \%$ Tween 20. Trehalose is a sugar that has been shown to preserve the activity of streptavidin ${ }^{26}$ and antibodies when dried so that denaturing does not cause loss of activity when rehydrated. ${ }^{27}$ Tween 20 is a detergent that serves as a more stringent washing agent and helps lower surface tension to increase infiltration of aqueous solutions into the pores. Detailed studies to optimize the preservative buffer were not conducted however future studies are planned to investigate the impact of Tween 20 concentration on receptor activity. 
Biotinylated anti-chicken IgG (H\&L) from a goat host was used as a control receptor. This B$\mathrm{Ab}$ was prepared and used under the same conditions as described above for the biotinylated anti-rabbit IgG.

The target molecule was whole rabbit $\operatorname{IgG}$ antibody (Ab). The stock solution was diluted to 1 $\mathrm{mg} \mathrm{ml}^{-1}$ in PBS and $15 \mu \mathrm{l}$ was applied to the chip within a humidity chamber for 1 hour to allow diffusion of the antibody through the pores. The solution was rinsed off with PBS buffer and soaked in $5 \mathrm{ml}$ of PBS buffer for $10 \mathrm{~min}$ on a rocker plate at room temperature to reduce non-specific binding.

\subsection{Blocking Streptavidin Biotin-Binding Sites (Preblocking)}

The effects of altering the avidity of SA were investigated to determine if steric crowding in the linking chemistry could be overcome to increase target detection sensitivity. This was accomplished by preblocking SA biotin-binding sites with biotin and analyzing the detection of receptor and target. Each SA has four equivalent binding sites for biotin; therefore, the molar concentration of biotin-binding sites was calculated by multiplying the SA molar concentration by four. The molar equivalent of biotin (244.3 Da) was added to bind to the desired number of SA biotin-binding sites in solution. For example, a 4:1 molar ratio of SA biotin-binding sites to biotin would result in 1 biotin-binding site being blocked on the SA. The mixture was allowed to equilibrate for 2 hours at room temperature before being added to the sensor. A control experiment confirmed that free biotin did not nonspecifically bind to the surface-linked biotin (no red shift in the reflectance spectrum was detected). This ensures that any red shift observed following exposure to a solution containing preblocked SA was entirely due to the binding of SA to the surface-immobilized biotin. All solutions were diluted in PBS buffer.

\subsection{Optical Detection in a Porous Silicon Biosensor}

A normal incident beam of white light (spot size of $\sim 13 \mathrm{~mm}^{2}$ ) was exposed to the sensor surface. The optical reflectance spectrum was measured using an Ocean Optics Spectrophotometer HR2000 with an optical resolution of $0.33 \mathrm{~nm}$ pixel $^{-1}$. All measurements were taken on dry samples following exposure and wash procedures as previously described. Prior to recording optical shift measurements the intensity of the incident white light was adjusted to correct for changes in microcavity \% reflection. This co-aligns the before and after spectra which facilitates straightforward measurement of the optical shift which is specific to binding interactions that take place within the porous matrix. Optical measurements were taken in three different locations on each sensor. Each experiment was repeated at least twice on two separate PSi sensors. All plots within this paper contain error bars that represent the standard deviation resulting from the measurements taken from multiple locations on different sensors. The methodology of taking differential measurements at each specific point reduces error that would otherwise be due to small variances in the optical spectrum pattern. Consistent data was obtained on multiple sensors with a coefficient of variance remaining less than $15 \%$. Nonlinear least squares curve fitting was performed with Origin 7.0.

\section{Results}

\subsection{Optimization of Linking Chemistry to Maximize IgG Target Detection}

Biotin Layer-The binding kinetics of biotin-SA are well characterized and steric crowding effects have been reported on planar surfaces using surface plasmon resonance (SPR). ${ }^{12}$ Recently, Ouyang et al. ${ }^{19}$ observed steric crowding when binding SA to biotin in a PSi optical sensor ${ }^{13}$, but the effect was not characterized in detail. In this study we characterize the effect of each layer in the sandwich assay on the immobilization of the subsequent layer with the goal of optimizing IgG target detection sensitivity. Starting from the bottom layer and moving upwards, the optical sensor response was first characterized following adsorption of amine 
reactive biotin (biotin-LC-LC-NHS) on APTMS treated surface. In Figure 3(a) a saturation curve can be seen for the optical reflectance red shift as a function of biotin solution concentration applied to the sensor. The fitted Langmuir adsorption curve (Fig 3(a)) begins to saturate at approximately $1.5 \mathrm{mg} \mathrm{ml}^{-1}$ biotin which is referred to as $100 \%$ biotin surface coverage. This is the maximum amount of biotin that bound to the internal PSi surface area. Based on a geometrical model of our device architecture ${ }^{28}$ we approximate the total internal surface area of the sensor to equal $\sim 26 \mathrm{~cm}^{2}$. A maximum red shift of $\sim 7.9 \mathrm{~nm}$ occurs at the $100 \%$ surface coverage of biotin. A $4.5 \mathrm{~nm}$ red shift indicates a surface coverage of $\sim 57 \%$ which results following the application of $0.5 \mathrm{mg} \mathrm{ml}^{-1}$ biotin-LC-LC-NHS. It can also be seen that zero red shift was observed following application of PBS buffer only.

SA-Biotin Linking Chemistry-The effect of biotin surface coverage on binding SA was studied next. The concentration of biotin-LC-LC-NHSsolution applied to the sensor was varied and the red shift associated with binding SA was measured for various SA concentrations $(0.1$, $0.4,0.8,1.0 \mathrm{mg} \mathrm{ml}^{-1}$ ). Results are plotted in Figure 3(b). Non-specific binding of SA was not observed $(0 \mathrm{~nm}$ red shift) in a control experiment conducted by applying streptavidin solution $\left(1 \mathrm{mg} \mathrm{ml}^{-1}\right)$ to an amino silanized sensor (no biotin). All four data sets in Figure 3(b) were fit to a Gaussian curve and the center $\mathrm{x}$-value was determined. For each SA solution the biotin concentration at which the maximum amount of streptavidin bound to the sensor (center $\mathrm{x}$ value of Gaussian) was $0.5 \pm 0.035 \mathrm{mg} \mathrm{ml}^{-1}$ biotin ( $57 \%$ surface coverage). The decrease in the amount of SA that binds at higher biotin surface coverage has been previously observed and attributed to steric crowding in the immobilized biotin layer. ${ }^{12,13,19}$ The initiation of this effect on porous silicon biosensors correlates well with that previously reported ${ }^{19}$. Our data however, is important in that it provides definitive evidence that steric crowding does indeed arise in the biotin layer as the observed decrease in SA binding is independent of the SA concentration applied to the sensor surface. Moreover, it seems plausible, given the topographical heterogeneity within the porous silicon matrix, that steric crowding would initiate at a higher (57\%) biotin surface coverage compared to that (20\%) observed on planar gold surfaces. ${ }^{12,13}$ (Refer to S1 for a schematic visualization.) It is also interesting to note that the SA binding approaches zero at high surface biotin coverage on porous silicon. This contrasts results found on planar surfaces where the SA binding asymptotesto $\sim 65 \%$ of maximum at high biotin coverage. ${ }^{12} \mathrm{We}$ attribute this difference to reduced pore infiltration of SA at high biotin coverage due to concentrated SA binding at the entrance of pores. In contrast to planar techniques, SA binding thatoccur s external of the porous matrix is not quantified in the linear red shift reported here.

Receptor Antibody Binding to SA-Biotin Complex-Next in the sandwich assay we analyzed the correlation between the streptavidin layer and the biotinylated receptor antibody (B-Ab). Measurements were made keeping the biotin coverage fixed at $57 \%\left(0.5 \mathrm{mg} \mathrm{ml}^{-1}\right)$ to maximize binding of the SA and avoid steric crowding in the biotin layer. It is important to note that for a fixed biotin surface coverage the binding of SA asymptotes with increasing surface coverage (Fig S2). In Figure 3(c) the B-Ab red shift $\left(0.5 \mathrm{mg} \mathrm{ml}^{-1}\right)$ is plotted for varying concentrations of SA applied to the sensor. Again, we observe a strong dependence of B-Ab binding on SA concentration. At SA concentrations higher than $70 \mu \mathrm{g} \mathrm{ml}^{-1}$ a sharp decrease in red shift (ie. magnitude of $\mathrm{B}-\mathrm{Ab}$ binding) is observed. The data again suggests that higher surface coverage of SA induces steric crowding in that layer, which hinders the amount of B$\mathrm{Ab}$ that can bind.

Target Binding \& Specificity of Linking Chemistry-Using the optimal concentrations as previously determined for both biotin $\left(0.5 \mathrm{mg} \mathrm{ml}^{-1}\right)$ and SA $\left(70 \mu \mathrm{g} \mathrm{ml}^{-1}\right)$, the concentration of biotinylated anti-rabbit IgG (receptor) was varied to determine its effect on capturing rabbit $\mathrm{IgG}$ (target). It can be seen in Figure 4(a) that the target detection peaks with a maximum of 
bound rabbit IgG at $70 \mu \mathrm{g} \mathrm{ml}^{-1}$ biotinylated anti-rabbit $\operatorname{IgG}$ (receptor) solution concentration. This will later be referred to as the optimal receptor concentration, which is coincidently equivalent to the optimum SA concentration. This concentration; however, corresponds to an immobilized receptor surface coverage $\sim 3$ times lower than SA, assuming thatall molecules bind (Fig S3).

Specificity of linking chemistry was investigated with the use of a biotinylated anti-chicken $\operatorname{IgG}$ (control receptor). Some non-specific binding was observed at low immobilized receptor concentrations (Fig 4a). This is believed to result from interaction of $\mathrm{IgG}$ with the positive amine terminated surface ${ }^{29}$ due to the fact that little cross-reactivity was observed between the control receptor and the target (rabbit IgG) in ELISA. At higher control receptor concentrations, target IgG is obstructed from the amine terminated surface and non-specific binding was diminished. Blocking agents could be employed to alter nonspecific binding, however in this study we chose to investigate steric crowding effects without interference from any blocking reagents.

Target Binding Kinetics and Detection Sensitivity-A kinetic study was completed to determine the soak time needed for optimum target signal detection on the developed PSi sensor with minimal steric crowding in each layer of the sandwich-based linking chemistry (biotin/SA/B-Ab). A solution containing a known amount of rabbit $\operatorname{IgG}\left(0.25 \mathrm{mg} \mathrm{ml}^{-1}\right)$ was applied to the sensor surface and the red shift was monitored over fixed intervals of time. Data shown in Figure 4(b) illustrates that target detection is possible within 10 minutes but that maximum signal results after approximately 45 minutes. The observed time for saturation in $\mathrm{IgG}$ association is consistent with previously published data of rabbit IgG binding to macroporous silicon Fabry-Perot single layers that were coated with protein A. ${ }^{30}$

A calibration curve was generated for the detection of rabbit IgG and is plotted in Figure 4(c). The reflectance spectrum red shift is plotted as a function of the concentration of rabbit IgG exposed to the PSi sensor for a soak time of 60 minutes (greater than the saturation time of $45 \mathrm{~min}$ ). It can be seen that the curve begins to saturate at a rabbit $\mathrm{IgG}$ concentration of $3 \mathrm{mg}$ $\mathrm{ml}^{-1}$. The linear range of rabbit IgG detection was found from $0.07-0.5 \mathrm{mg} \mathrm{ml}^{-1}$. Lower concentrations of rabbit IgG are measurable, however shifts $<0.7 \mathrm{~nm}$ can not be measured with certainty given the wavelength resolution of the spectrophotometer. Knowing that the wavelength shift sensitivity $(\Delta \lambda / \Delta \eta)$ of our device is $140 \mathrm{~nm} / \mathrm{RI}$ and that PSi has a linear response to change in its dielectric environment ${ }^{21}$, it can be determined that a $1 \mathrm{~nm}$ red shift is analogous to a $\Delta \eta$ of 0.005 . While methods to improve the lower limit of detection are underway this current sensitivity range is sufficient for the detection of specific IgG in blood $\left(\sim 6-10 \mathrm{mg} \mathrm{ml}^{-1}\right)^{31}$.

\subsection{Manipulating the SA-biotin Link}

In an effort to enhance target detection sensitivity we explored the possibility of manipulating the SA linkage to mitigate the effects of steric crowding in that layer. Streptavidin has four equivalent binding sites for biotin with twoon each half of the molecule. ${ }^{1,32}$ The number of free biotin-binding sites on SA was varied by adding molar equivalents of biotin in solution and letting that stabilize prior to applying the preblocked SA solution to the biotinylated sensor surface. B-Ab binding to surface-linked SA as a function of biotin surface coverage and the number of free biotin-binding sites (preblocking) on SA was investigated to gain insight to the binding behavior of SA and how this layer effects $\mathrm{B}-\mathrm{Ab}$ receptor immobilization.

Biotinylated Antibody Receptor (B-Ab) Binding Behavior with Preblocking-We measured the magnitude of B-Ab red shift $\left(0.5 \mathrm{mg} \mathrm{m}^{-1}\right)$ as a function of SA concentration for low $(10 \%)$, medium (57\%), and high (90\%) biotin surface-coverage (Fig 5). Pérez-Luna et 
al. ${ }^{13}$ showed that SA bound to surface linked biotin via two links at the biotin coverage where steric crowding was observed to initiate on planar surfaces. Consequently on our heterogeneous surface, SA could be assumed to bind to $57 \%$ biotin surface-coverage via two links. (Data supporting this assumption on PSi is shown in Fig S8.) Therefore; with 2 preblocked biotinbinding sites (PB-BBS) on SA a precipitous decrease in B-Ab red shift is anticipated for $57 \%$ biotin surface-coverage as there will be no free sites remaining to attach the B-Ab receptor.

Figure 5 displays the results for 0 and 2 PB-BBS on SA from which definitive trends are apparent. At high (90\%) biotin surface coverage, steric crowding significantly hinders SA binding (Fig 3(b)) due to steric constraints introduced by the close proximity of the surfacelinked biotin. SA is most likely restricted to binding to surface immobilized biotin at the edges of biotin islands ${ }^{33}$ or at peaks associated with the heterogeneous topography of the PSi (illustrated in S1). With minimal immobilized SA, little B-Ab can specifically bind to the sensor, resulting in little difference between B-Ab binding to SA with 0 or 2 PB-BBS (Fig 5 (c) and (f) respectively).

The greatest change in B-Ab binding with preblocking SA is found at medium (57\%) biotin surface coverage (Fig 5) as expected. This is due to steric crowding initiating in the biotin layer (Fig 3(b)) and the assumption that SA is more likely to bind via two surface biotin links. When comparing the plots for B-Ab binding at $57 \%$ biotin surface coverage with 0 and 2 PB-BBS (Fig 5 (b) and (e)) we first observe a notable decrease in the peak magnitude of the red shift. With 2 PB-BBS any SA bound via 2 links cannot bind B-Ab. Therefore, the B-Ab binding observed (Fig 5(e)) is restricted to SA bound to the biotin via 1 link. The sparse distribution of available binding sites for B-Ab has a positive effect in alleviating steric crowding which would otherwise reduce $\mathrm{B}-\mathrm{Ab}$ binding. This reduction in the influence of steric crowding with 2 PB-BBS is shown by an increase in B-Ab binding for SA concentrations $>0.4 \mathrm{mg} \mathrm{ml}^{-1}$ (Fig 5(e)). It is of interest to note that two different biotinylated receptor antibodies (chicken and rabbit) were used to generate the data presented in Figures 5(b) and (e) and both antibodies exhibited the same trends. This suggests that behaviors exhibited by this system are applicable to any biotinylated antibody receptor used with the SA/biotin linking chemistry.

At low (10\%) biotin surface coverage, steric crowding in the biotin layer is less prevalent. Assuming that SA is mainly bound to surface linked biotin via 1 link at this low (10\%) biotin surface coverage there is 1 free biotin-binding site left on the SA with 2 PB-BBS (Fig 5(d)). The remaining free biotin-binding site can be in two configurations: (1.) one free biotin-binding site outward (free to bind $\mathrm{B}-\mathrm{Ab}$ ) and (2.) one free biotin-binding site inward towards surface (not free to bind B-Ab). A mix of these two configurations on the surface would result in forcing more space between bound B-Ab and influence steric crowding effects. Due to the fact that SA molecules are already sparsely distributed at low biotin coverage alleviation of steric crowding has less impact, therefore the shape of the curve did not change as drastically seen with higher biotin coverage (Fig 5(d)). A delay in the onset of steric crowding is observed by the peak in the B-Ab binding curve occurring at a higher SA concentration $(0.1 \mathrm{mg} \mathrm{ml}-1 \mathrm{vs}$. $0.07 \mathrm{mg} \mathrm{ml}^{-1}$ ), resulting in a small increase of B-Ab receptor immobilization ( $4.8 \mathrm{~nm}$ vs. 3.4 $\mathrm{nm}$ red shift, Fig 5(d) vs. Fig 5(a)).

This data gives evidence through consistent trends that preblocking SA does indeed reduce steric crowding effects within the SA layer which translates into increases in B-Ab binding. Unfortunately the increases observed with preblocking were insufficient to enhance receptor immobilization beyond the maximum achieved at $57 \%$ biotin surface coverage with no preblocking (Fig 5(b)). Other middle range biotin surface coverage values (37\% and $65 \%$ ) were investigated with 2PB-BBS and slight increases in receptor (B-Ab) binding were found (data not shown), however the total immobilized receptor concentrations again did not improve upon what was found with no preblocking at $57 \%$ biotin surface coverage. In a final effort to 
enhance B-Ab receptor immobilization 1 PB-BBS was tested on low (10\%) biotin surface coverage (Fig. S5) and medium biotin surface coverage (57\%, data not shown). Again, only slight increases in B-Ab binding were observed and this strategy of preblocking to enhance receptor immobilization was no longer pursued.

Preblocking SA Final Effects on Target Detection-In a complex sandwich assay, such as the one employed here, we have shown that each layer has an effect on the final target detection. The slight increase in B-Ab receptor binding that was observed due to preblocking SA (Fig 5(d) \& Fig S5) showed promise of reducing some of the negative effects induced by steric crowding in the SA layer. Unfortunately preblocking did not result in higher surface coverage of receptor than was achieved at $57 \%$ biotin surface coverage with no preblocking (Fig 5(b) $\sim 8.6 \mathrm{~nm}$ red shift). Hence the application of preblocking would not be expected to enhance target detection. This was tested and confirmed by analyzing the detection of target (rabbit IgG) on a sensor with $10 \%$ biotin surface coverage and SA $\left(0.1 \mathrm{mg} \mathrm{ml}^{-1}\right)$ with 2 PBBBS for varying concentrations of immobilized receptor (Fig S6). Results showed a little less than half of the sensitivity in detecting target $\left(1 \mathrm{mg} \mathrm{ml}^{-1}\right.$ rabbit IgG) when compared to the optimized linking chemistry at $57 \%$ biotin surface coverage with no preblocking. (Fig 4(a)).

\section{Conclusion}

We succeeded in developing a macroporous silicon optical biosensor to conduct immuno-based sandwich assay in a label free format. It was demonstrated through a "bottom-up" approach that steric crowding occurs in each layer (biotin, SA, and receptor antibody) of the sandwich assay (Figs 3-4). Hence, the surface coverage of each layer must be precisely controlled to achieve optimum target detection. While the effect of steric crowding in the biotin layer on binding streptavidin was previously known, we report here that surface topography delays the onset relative to planar surfaces. Moreover, this study is the first to systematically investigate in detail how the effects of steric crowding translate up into each layer of the sandwich assay.

To overcome steric crowding effects and to elucidate its impact on target detection we manipulated the avidity of the SA link by preblocking in hopes that this could develop into a general methodological approach to optimize the use of the streptavidin link in various biotechnology applications. Our results showed (Fig 5) that altering the avidity of the SA does induce changes in steric crowding within that layer resulting in improved receptor immobilization. This improvement however, was not profound enough to increase receptor immobilization beyond what was achieved by the optimized linking chemistry with no preblocking.

The detection sensitivity range of rabbit IgG demonstrated in this study $\left(0.07-3 \mathrm{mg} \mathrm{ml}^{-1}\right)$ is adequate for measuring specific changes in IgG blood concentrations. To achieve facile infiltration of biomacromolecules into porous matrix the device must be fabricated from macroporous PSi (pore diameters $>50 \mathrm{~nm}$ ). 7,21,34,35,36 However, large pore devices typically have lower porosity and consequently exhibit a lower wavelength shift sensitivity as is observed in this study. ${ }^{7}$ Several approaches are currently being explored to improve detection sensitivity of macroporous PSi sensors including: improving the spectrophotometer wavelength resolution, fabricating devices to operate further in the NIR, and optimizing the PSi device to obtain maximum porosity while enabling pore infiltration of macromolecules. In conclusion, the insight obtained by this in depth study of steric crowding effects on detection sensitivity can be translated to many biotechnology applications employing the biotin/SA linking chemistry on heterogeneous topography. 


\section{Supplementary Material}

Refer to Web version on PubMed Central for supplementary material.

\section{Acknowledgements}

The authors graciously acknowledge the NIH / NIAID 5K25AI060884-02 for financial support of this research, Benjamin Miller for insightful discussions about biosensing and Nicholas Winograd his help regarding the use of trehalose.

\section{References}

1. González M, Gagatolli LA, Echabe I, Arrondo JLR, Argarańa CE, Cantor CR, Fidelio GD. J. of Biol. Chem 1997;272:11288-11294. [PubMed: 9111033]

2(a). Sohn H, Letant S, Sailor MJ, Trogler WC. J. Am. Chem. Soc 2000;122:5399-5340. (b) Chan S, Horner SR, Miller BL, Fauchet PM. J. Am. Chem. Soc 2001;123:11797-11798. [PubMed: 11716737]

3. Haes AJ, Van Duyne RP. J. Am. Chem. Soc 2002;124:10596-10604. [PubMed: 12197762]

4. Rodgers JT, Patel P, Hennes JL, Bolognia SL, Macotti DP. Anal. Biochem 2000;277:254-259. [PubMed: 10625515]

5. Gruber HJ, Marek M, Schindler H, Kaiser K. Bioconjugate Chem 1997;8:552-559.

6. Takeshi S, Cantor CR. J. of Bio. Chem 1990;265:3369-3373. [PubMed: 2406253]

7. DeLouise LA, Kou PM, Miller BL. Anal. Chem 2005;77:3222-3230. [PubMed: 15889912]

8. Kholodenko BN. Nature Reviews Mol. Cell Bio 2006;7:165-176.

9. Kornberg L, Earp HS, Parsons JT, Schaller M, Juliano RL. J. of Bio. Chem 1992;267:23439-23442. [PubMed: 1429685]

10. Betozzil CR, Kiessling LL. Science 2001;291:2357-2364. [PubMed: 11269316]

11. Puls I, Jonnakuty C, LaMonte BH, Holzbaur EL, Tokito M, Mann E, Floeter MK, Bidus K, Drayna D, Oh SJ, Brown RH Jr. Ludlow CL, Fischbeck KH. Nat Genet 2003;33(4):455-456. [PubMed: 12627231]

12. Jung LS, Nelson Kjell E. Stayton PS, Campbell CT. Langmuir 2000;16:9421-9432.

13. Pérez-Luna VH, O’Brien MJ, Opperman KA, Hampton PD, Lòpez GP, Klumb LA, Stayton PS. J. Am. Chem. Soc 1999;121:6469-6478.

14. Deckert AA, Farrell C, Roos J, Waddell R, Stubna A. Langmuir 1999;15:5578-5583.

15. Prasad A, Balakrishnan S, Jain SK, Jain GC. J. Electrochem. Soc 1982;129(3):596-599.

16. Lysenko V, Bidault F, Alekseev S, Zaitsev V, Barbier D, Turpin C, Geobaldo F, Rivolo P, Garrone E. J. Phys. Chem. B 2005;109(42):19711-19718. [PubMed: 16853549]

17. Schwartz MP, Cunin F, Cheung RW, Sailor MJ. physica status solidi a 2005;202(8):1380-1384.

18. Vinegoni, C.; Cazzanelli, M.; Pavesi, L. Silicon-Based Materials and Devices. Nalwa, HS., editor. 2. Academic Press; New York: 2001. p. 124-188.

19. Ouyang H, Christophersen M, Viard R, Miller BL, Fauchet PM. Adv. Funct. Mater 2005;15:18511859.

20 (a). Dancil KS, Greiner DP, Sailor MJ. J. Am. Chem. Soc 1999;121:7925-7930. (b) DeLouise LA, Miller BL. Anal Chem 2004;76:6915-6920. [PubMed: 15571341]

21. Bruggeman DAG. Ann. Phys 1925;24:636.

22. Taylor AE, Parker JC. J. of Physiology 2003;553:333.

23. Janshoff, Dancil K-PS, Steinem C, Greiner DP, Lin VS-Y, Gurtner C, Motesharei K, Sailor MJ, Ghadiri MR. J. Am. Chem. Soc 1998;120:12108.

24. Steinem C, Janshoff A, Lin VS-Y, Völcker NH, Ghadiri MR. Tetrahedron 2004;60:11259-11267.

25. Wang X, Wnchuang H, Ramasubramaniam R, Bernstein G, Snider G, Lieberman M. Langmuir 2003;19:9748-9758.

26. Nan X, Shumaker-Parry JS, Zareie MH, Campbell CT, Castner DG. Langmuir 2004;20:3710-3716. [PubMed: 15875404] 
27. Jairo J, Michel P. Current Medicinal Chem 2004;11(8):439-446.

28. DeLousie LA, Miller BL. Anal. Chem 2004;76:6915-6920. [PubMed: 15571341]

29. Graves HC. J Immunol Methods 1988;111:157-166. [PubMed: 2456353]

30. Schwartz MP, Alvarex SD, Sailor MJ. Anal. Chm 2007;19(2007):327-334.

31 (a). Weisbroth, SH.; Flatt, RE.; Kraus, AL. The Biology of the Laboratory Rabbit. Academic Press; New York, NY: 1974. p. 62-64. (b) Butler WT, Rossen RD. J. Clin. Invest 1973;52:2629-2640. [PubMed: 4729056]

32. Avrantinis SK, Stafford RL, Tian X, Weiss GA. Chem. Biochem 2002;3:1229-1234.

33. Doudevski I, Schwartz DK. J. Phys. Chem. B 2000;104:9044-9047.

34. Tinsley-Bown AM, Canham LT, Hollings M, Anderson MH, Reeves CL, Cox TI, Nicklin S, Squirrell DJ, Perkins E, Hutchinson A, Sailor MJ, Wun A. Phys. Status Solidi A 2000;182:547-553.

35. Arwin H, Gavutis M, Gustafsson J, Schultzberg M, Zangooie S, Tengvall P. Physica Status Solidi A 2000;182:515-520.

36. Karlsson LM, Tengvall P, Lundström I, Arwin H. Phys. Status Solidi A 2003;197:326-330. 

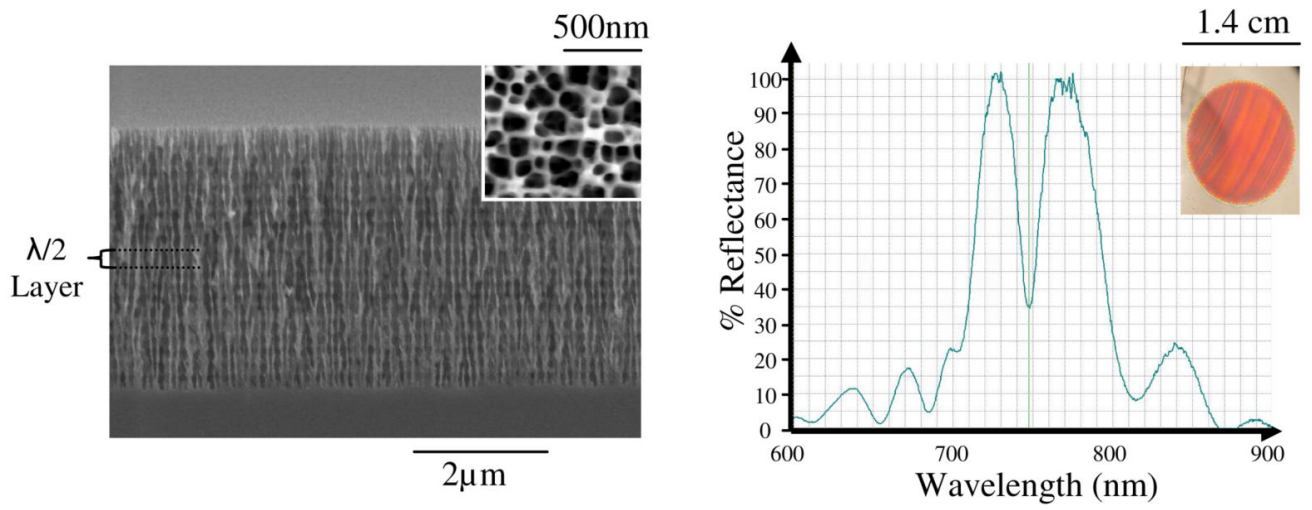

Figure 1.

(a) SEM micrograph cross section of $\mathrm{n}^{+}$microcavity; top view shown in inset. (b) Optical reflectance spectrum of microcavity; picture of the PSi biosensor shown in inset. 


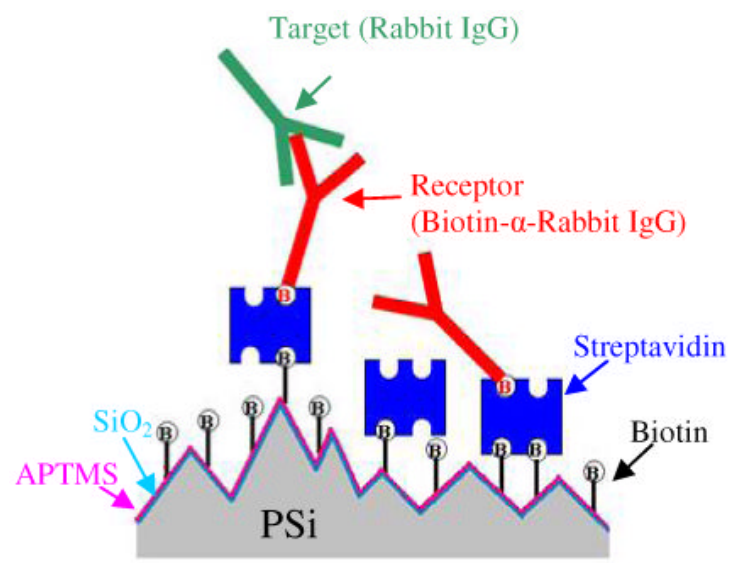

Figure 2.

Schematic illustrating linking chemistry, receptor, and target molecules binding on topographically heterogeneous porous silicon substrate 


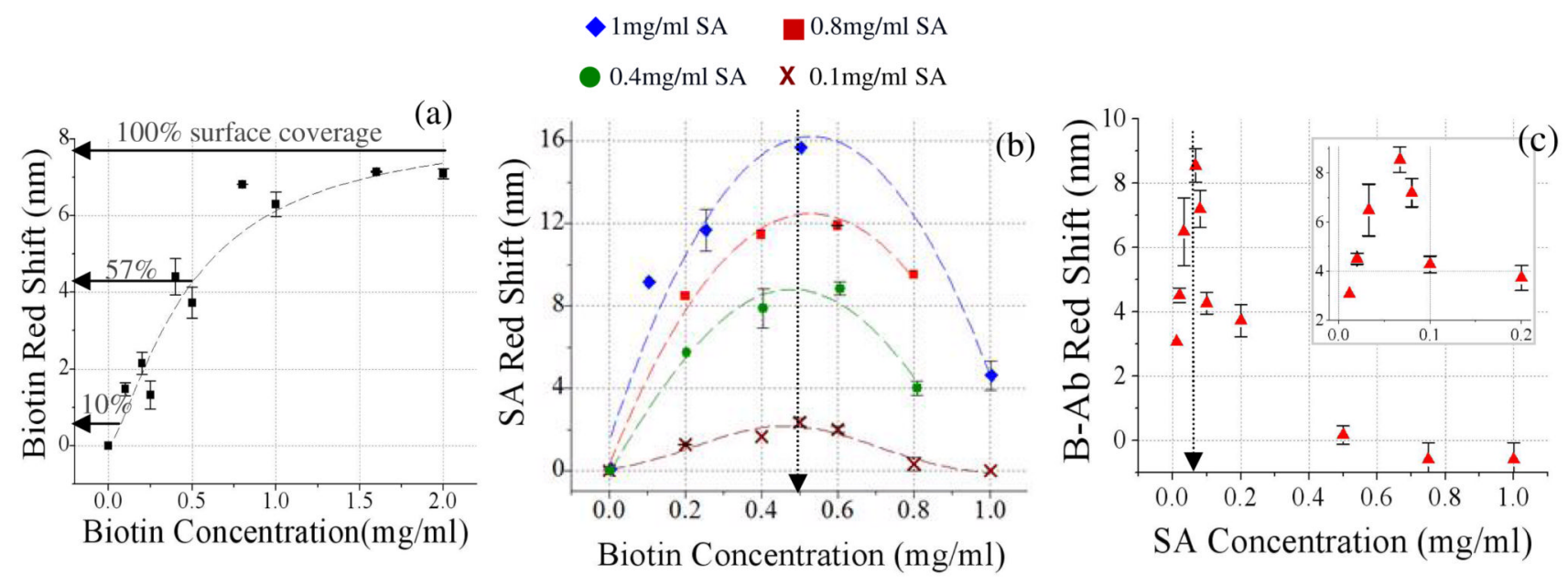

Figure 3.

(a) Biotin-LC-LC-NHS binding curve per concentration applied to sensor. (b) Red shift due to binding of SA for varying concentrations of biotin-LC-LC-NHS applied to sensor surface. Family of four curves due to various SA concentrations $(0.1,0.4,0.8,1.0 \mathrm{mg} / \mathrm{ml})$ applied to sensor. (c) Red shift due to binding of biotinylated antibody receptor for varying concentrations of SA applied to sensor. (Biotin-LC-LC-NHS=0.5mg/ml, B- $\alpha$-rabbit $\mathrm{IgG}=0.5 \mathrm{mg} \mathrm{ml}^{-1}$ ) 

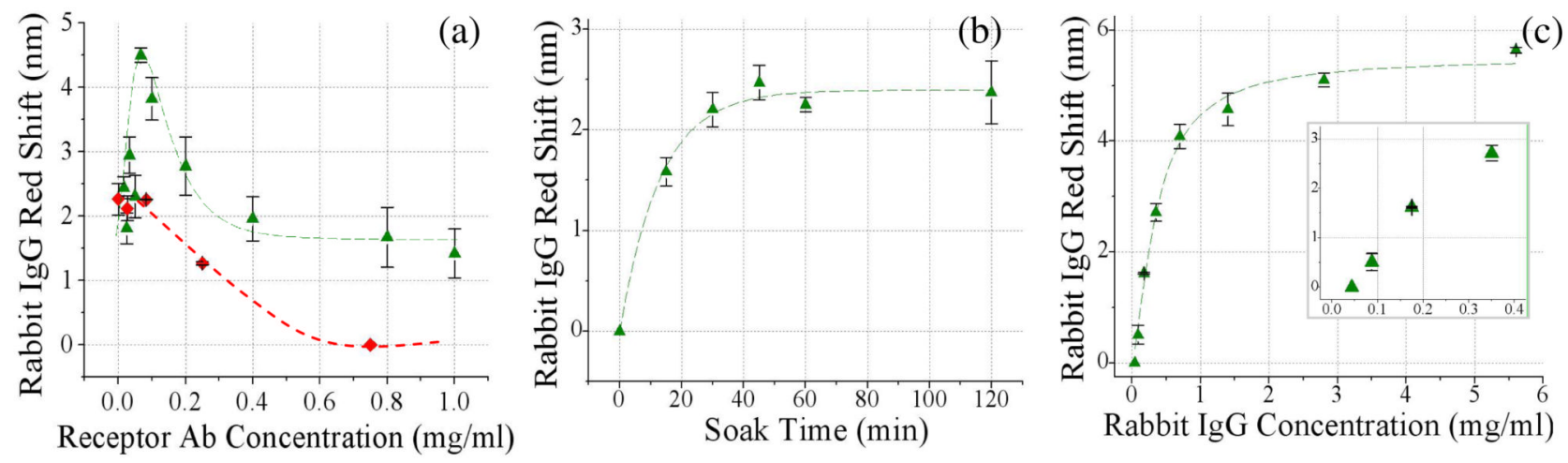

Figure 4.

(a) Specific detection of target rabbit $\operatorname{IgG}\left(1 \mathrm{mg} \mathrm{ml}^{-1}\right)$ with a biotinylated anti-rabbit $\operatorname{IgG}$ receptor (green triangle) and non-specific binding of rabbit IgG to biotinylated anti-chicken $\mathrm{IgG}$ receptor (red diamond) for varying concentrations of receptor molecules. ([Biotin-LC-LCNHS $]=0.5 \mathrm{mg} \mathrm{ml}^{-1}$; [SA] $\left.=0.07 \mathrm{mg} \mathrm{ml}^{-1}\right)$. (b) Time study of rabbit $\mathrm{IgG}\left(0.25 \mathrm{mg} \mathrm{ml}^{-1}\right)$ binding to optimized linking chemistry (biotin-LC-LC-NHS=0.5 mg ml${ }^{-1}, \mathrm{SA}=0.07 \mathrm{mg} \mathrm{ml}^{-1}$, B$\mathrm{Ab}=0.07 \mathrm{mg} \mathrm{ml}^{-1}$ ). (c) Rabbit IgG calibration curve (biotin-LC-LC-NHS=0.5 mg ml-1, $\mathrm{SA}=0.07 \mathrm{mg} \mathrm{ml}^{-1}, \mathrm{~B}-\mathrm{Ab}=0.07 \mathrm{mg} \mathrm{ml}^{-1}$, rabbit IgG concentration varied) (a) - (c) All concentrations refer to solution concentrations applied to sensor. 

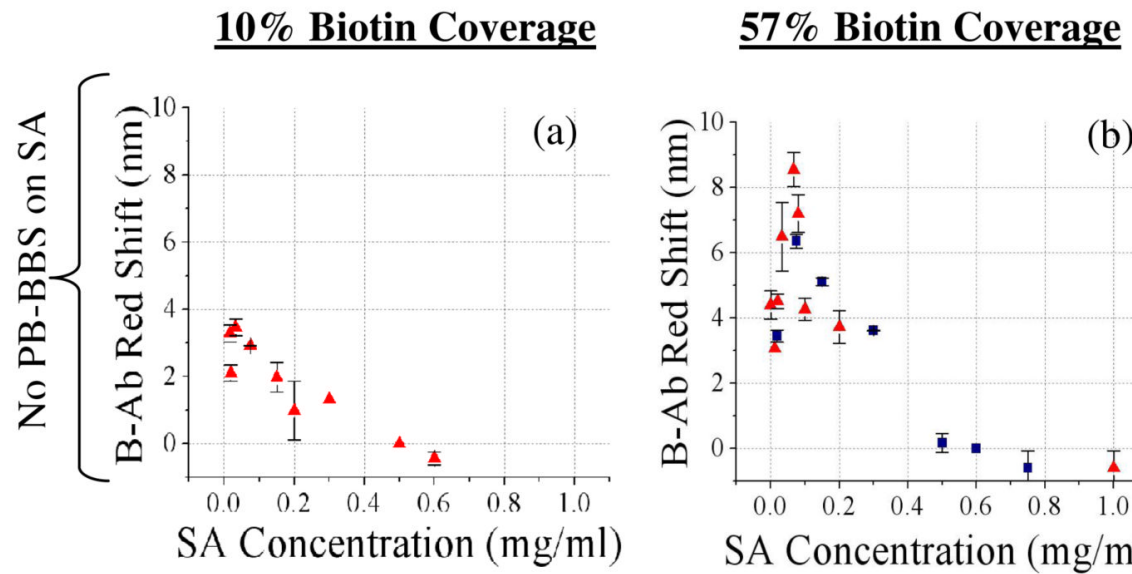

90\% Biotin Coverage
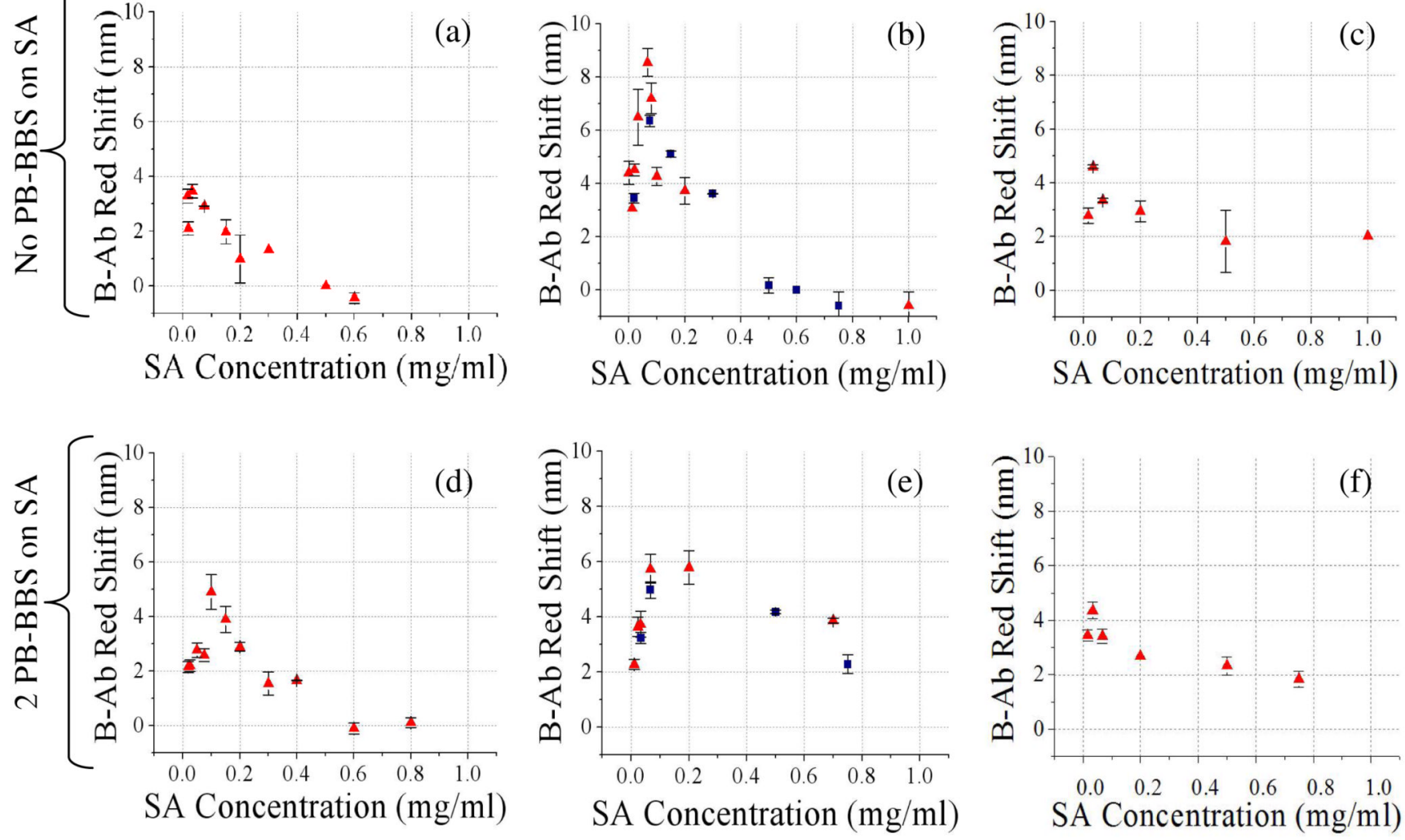

Figure 5.

Biotinylated Ab red shift as a function of varying SA concentration. ([B-Ab] $=0.5 \mathrm{mg} \mathrm{ml}^{-1}$ ) No preblocked biotin-binding sites (PB-BBS) on SA at (a) $10 \%$ biotin surface coverage, (b) 57 $\%$ biotin surface coverage (repeated in 5 experiments), (c) $90 \%$ biotin surface coverage. 2 PBBBS on SA at: (d) $10 \%$ biotin surface coverage, (e) $57 \%$ biotin surface coverage (repeated in 3 experiments), (f) $90 \%$ biotin surface coverage. (Red triangles = biotinylated $\alpha$-rabbit IgG receptor, blue squares $=$ biotinylated $\alpha$-chicken IgG control receptor) (a)-(f) All concentrations refer to 